\title{
Asthma-chronic obstructive pulmonary disease overlap syndrome in Poland. Findings of an epidemiological study
}

\author{
Dorota Brzostek, Marek Kokot
}

Takeda Poland

Postep Derm Alergol 2014; XXXI, 6: 372-379

DOI: 10.5114/pdia.2014.47120

\begin{abstract}
Introduction: Recent years have seen an increased interest in asthma-chronic obstructive pulmonary disease (COPD) overlap syndrome (ACOS).

Aim: In 2012, Takeda Polska conducted a non-interventional epidemiological study aimed at identifying the typical phenotype of ACOS patients receiving pulmonary care.

Material and methods: The study enrolled a total of 12,103 of smoking patients above 45 years of age (mean age: 61.5 years; mean duration of smoking: 28.4 pack-years). A total of $68.6 \%$ of patients represented the frequentexacerbation phenotype (mean number of exacerbations during 12 months: 2.11 ), and $56.4 \%$ of patients from the group comprising 12,103 participants were hospitalized at least once during their lifetime due to a respiratory system disease (mean number: $3.82 \pm 3.76$ ).

Results: The most commonly found asthma symptoms included paroxysmal dyspnoea with wheezing, and good response to inhaled steroids. The most frequently identified COPD-associated symptoms were: long-lasting reduction in forced expiratory volume in $1 \mathrm{~s}\left(\mathrm{FEV}_{1}\right)(<80 \%$ after administering a bronchodilator) and chronic productive cough. Eighty-five percent of patients were diagnosed with concomitant diseases, predominantly arterial hypertension (62.9\%) and metabolic diseases (metabolic syndrome, obesity, type 2 diabetes $-46.4 \%$ in total).

Conclusions: A clinically severe course of ACOS and the presence of concomitant diseases should be regarded as factors justifying an individual selection of inhalation therapy which specifically takes into account anti-inflammatory treatment and patient safety.
\end{abstract}

Key words: asthma, chronic obstructive pulmonary disease, asthma-chronic obstructive pulmonary disease overlap syndrome, concomitant diseases, phenotype.

\section{Introduction}

In recent years, attention has been drawn to the fact that some patients with asthma or chronic obstructive pulmonary disease (COPD) cannot be unambiguously qualified as having one of the enumerated syndromes on the basis of definitions laid down by the most widely accepted international guidelines, i.e. GINA and GOLD, respectively. An analysis of such cases has revealed that they represent a mixed phenotype with a combination of symptoms of asthma and COPD. The findings have, in turn, lead to a revival of claims suggesting that there is a separate syndrome combining the traits of asthma and COPD. The syndrome does not have a specific name - it cannot really be recognized as a separate disease entity - so the term of "asthma-chronic obstructive pulmonary disease overlap syndrome" (ACOS) has been proposed. It is not known whether asthma and COPD are diseases with different pathogeneses ("British theory") or share a common pathogenetic pathway ("Dutch theory") or perhaps, as has recently been suggested, an additional pathomechanism, specific only to the overlap syndrome, is implicated [1]. Since there are no universally recognized criteria for diagnosing the syndrome, the profile of patients classified as suffering from ACOS is markedly different across the studies, depending on the adopted rules of study enrolment. One observation, however, seems to be common to the majority of studies: the fact that ACOS patients tend to have a severe course of the disease, with more symptoms, more exacerbations and more frequent hospitalizations, and a poorer prognosis - perhaps due to the more frequent presence of concomitant diseases, including metabolic and circulatory disorders [2, 3]. They are patients suffering from a severe general medical condition in whom, to aggravate the situation, COPD treatment is more difficult and less effective, and the prognosis as regards disease stabilization (management of

Address for correspondence: Marek Kokot, Takeda Poland, Al. Jerozolimskie 146 A, 02-305 Warsaw, Poland, phone: +48 2260813 00, phone/fax: +48 2260813 03, e-mail: marek.kokot@takeda.com Received: 22.10.2014, accepted: 25.11.2014. 
the disease understood as the lack of exacerbations) is essentially unfavourable. The systematization of diagnostic criteria was first undertaken by Spanish experts [4] in 2012. Along with a growing interest in the topic, and an increasing number of publications, new GINA guidelines (published on 6 May 2014) [5] incorporated a consensus statement on the overlap syndrome prepared by GINA and GOLD. In 2012, Takeda Polska conducted a cross sectional epidemiological study with a view to investigating the diagnosis and incidence of ACOS in the population of Polish patients treated by pulmonology specialists, and the profile (clinical phenotype) of patients diagnosed simultaneously with asthma and COPD.

\section{Aim}

The aim of the study was to describe the most common clinical features and results of additional examinations constituting the phenotype of a patient diagnosed by a medical specialist simultaneously with asthma and COPD, currently termed as "asthma and COPD overlap syndrome" (ACOS). The area of interest of the study also comprised the presence and types of concomitant diseases.

\section{Material and methods}

The study was conducted using a specially developed questionnaire which was identical all over Poland. The study involved 384 pulmonary specialists who enrolled a total of 12,103 patients in the period from March 2012 to September 2013. The study had a non-interventional design: data were collected during routine medical check-ups. According to the definition of a non-interventional study, participation did not require the physicians to perform any additional procedures aside from a routine medical check-up. During the medical appointment, the specialists enrolled patients in the study which consisted of the completion of a questionnaire by physician describing the course of the disease and the treatment currently received by the patients.

Only smoking patients (either current or past smokers) aged over 46 years were enrolled in the study a priori; nonsmokers were not enrolled. One physician was allowed to enrol a maximum of 32 patients under his/her consultation; one patient could only be enrolled once in the study.

The logistics of the study and the statistical processing of results for Takeda were provided by Quality Audit House (QAH), Lodz, Poland.

\section{Results}

The analysis covered a total of 12,103 patients at a mean age of $61 \pm 9.3$ years, including $46.9 \%$ of women and $53.1 \%$ of men (Figure 1). In accordance with the enrolment criterion, all the participants of the study were smokers. The mean intensity of the smoking habit was $28.4 \pm 13.5$ pack-years (Table 1 ).

\section{Clinical characteristics - elements determining the diagnosis of asthma and chronic obstructive pulmonary disease}

The assessment of the clinical profile of patients in the questionnaire was based on a list of clinical parameters for asthma and COPD - characteristic elements of medical history, including response to treatment, results of physical examination and typical laboratory tests. The questionnaire was based on the most common disease parameters used in medical practice without drawing on concurrently available literature indications (ACOS consensus - Soler-Cataluna, review studies by Louie et al.) (Table 2, Figures 2, 3).

The presence of clinical symptoms, including paroxysmal dyspnoea, was determined by the pulmonologist on the basis of interviews about the disease course. Paroxysmal dyspnoea did not exclude progressive exertional dyspnoea related with persistent obstruction (as presented in Table 2), typical parameters of asthma and COPD were assessed independently.

According to criteria of a simplified questionnaire adopted (Table 2), the obstruction is assessed only as FEV $<80 \%$ after administering a bronchodilator. Data concerning $\mathrm{FEV}_{1} / \mathrm{FVC}$, which should be $<0.7$ to confirm the obstruction were not collected. Patients with higher or lower degree of $\mathrm{FEV}_{1}$ reduction have not been assessed separately.

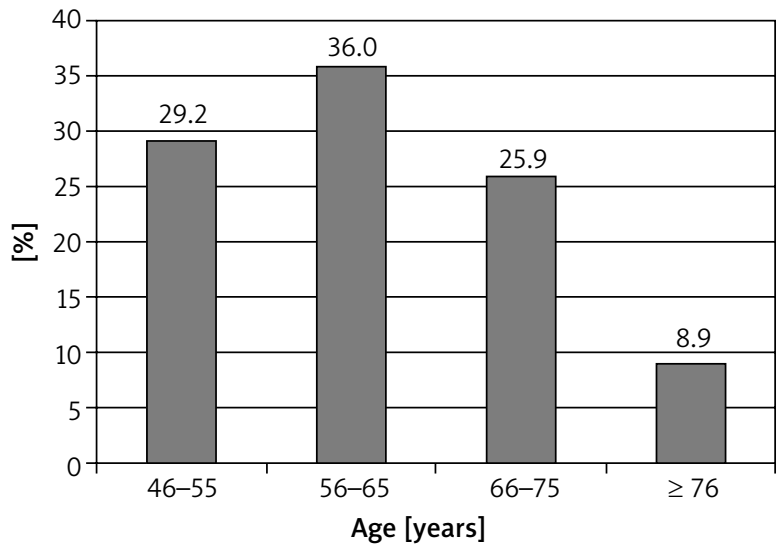

Figure 1. Patient demographics

Table 1 . Smoking in the study population $(n=12,103)$

\begin{tabular}{cc}
\hline Current smokers & Past smokers \\
\hline $42.6 \%$ & $57.4 \%$ \\
\hline \multicolumn{2}{c}{ Pack-year statistics } \\
\hline Mean & 28.42 \\
\hline Median & 29 \\
\hline Dominant & 30 \\
\hline Standard deviation & 13.45
\end{tabular}


Table 2. Criteria for the diagnosis of asthma and COPD noted in the study questionnaire

\begin{tabular}{|c|c|}
\hline Asthma & COPD \\
\hline - Family history of atopy & - Persistent reduction in $\mathrm{FEV}_{1}<80 \%$ (after administering a bronchodilator) \\
\hline - $\operatorname{lgE}>100 \mathrm{IU}$ & - Chronic productive cough \\
\hline - Positive skin-test results & - Clinical signs of emphysema \\
\hline - Other allergic symptoms & - Signs of lung hyperinflation in functional tests \\
\hline - Blood eosinophilia & - Signs of decreased lung density in HRCT \\
\hline - Sputum eosinophilia & - Progressive exertional dyspnoea \\
\hline - Variability of obstruction & - Signs of pulmonary heart syndrome in ECG or X-ray - diagnosed at any time \\
\hline - Good response to ICS & - Respiratory failure diagnosed at any time outside of an exacerbation \\
\hline - Early-onset bronchial disease & - Poor response to ICS \\
\hline \multicolumn{2}{|l|}{ - Paroxysmal unproductive cough } \\
\hline \multicolumn{2}{|c|}{ - Paroxysmal dyspnoea with wheezing } \\
\hline \multicolumn{2}{|l|}{ - Normal X-ray findings } \\
\hline \multicolumn{2}{|l|}{ - Normal PEF between attacks } \\
\hline - Others, please specify & \\
\hline
\end{tabular}

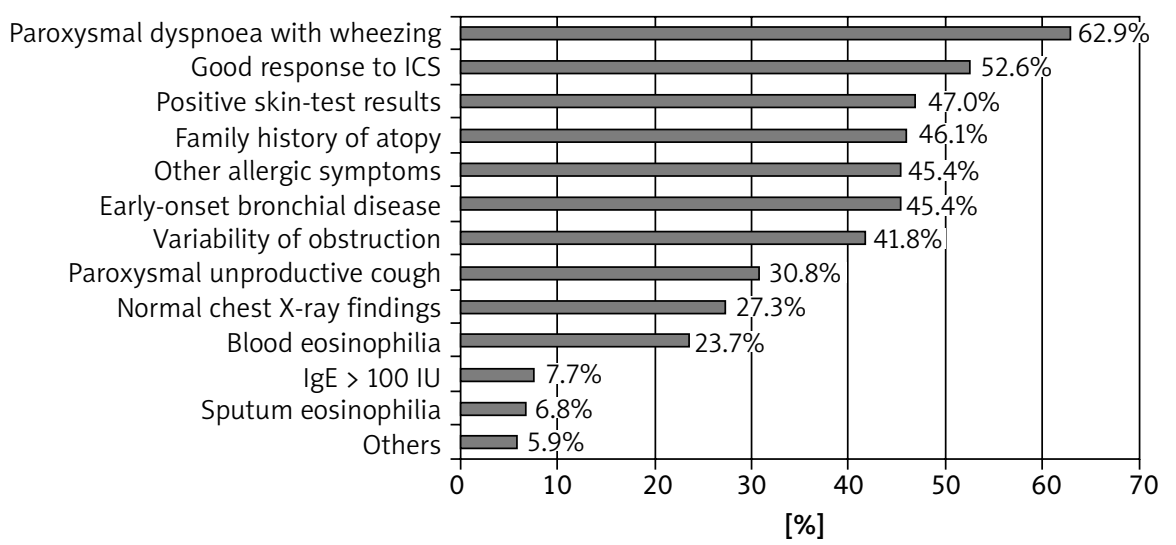

Figure 2. Most commonly found features typical of bronchial asthma (multiple response chart) $(n=12,103)$

Persistent reduction in $\mathrm{FEV}_{1}<80 \%$ (after administering a bronchodilator) Chronic productive cough

Progressive exertional dyspnoea

Clinical signs of emphysema

Signs of lung hyperinflation in functional tests

Signs of pulmonary heart syndrome in ECG or X-ray - diagnosed at any time

Respiratory failure diagnosed at any time outside of an exacerbation

Signs of decreased lung density in HRCT

Poor response to ICS

Others

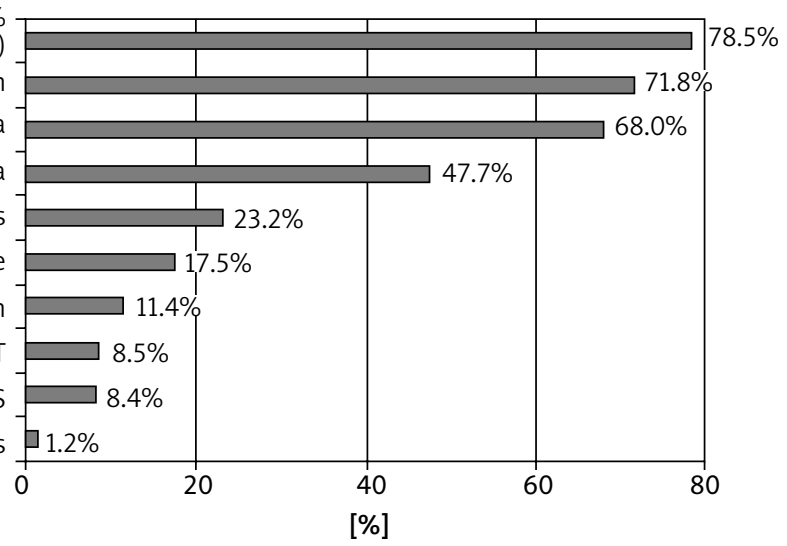

Figure 3. Most commonly found features typical of COPD (multiple response chart) $(n=12,103)$ 
Table 3. Exacerbations of the disease treated on an outpatient basis over the last 12 months of follow-up in the study population $(n=12,103)$

\begin{tabular}{cc}
\hline Patient had an exacerbation & Patient had no exacerbations \\
\hline $68.6 \%$ & $31.4 \%$ \\
\hline \multicolumn{2}{c}{ Exacerbation statistics } \\
\hline Mean & 2.11 \\
\hline Median & 2 \\
\hline Dominant & 1 \\
\hline Standard deviation & 1.76 \\
\hline
\end{tabular}

The most common criteria for diagnosing the asthma component in the study population included the history of paroxysmal dyspnoea with wheezing $(62.9 \%$ of the study patients) and previous good response to inhaled steroids (52.6\%). These criteria were followed by features of the patients' allergic history (positive skin-test results, family history of atopy, other allergic conditions and symptoms) and early-onset bronchial disease. The variability of obstruction also played a significant role (41.8\%) for the patients. Results of additional examinations, such as chest X-ray, blood eosinophil count, concentration of immunoglobulin E (IgE) and sputum eosinophilia, were less important for the diagnosis.

In contrast to asthma, the most crucial criterion of COPD diagnosis was persistent bronchial obstruction (78.5\% of patients). Other traits assisting in the diagnosis were: chronic productive cough (71.8\%) and progressive exertional dyspnoea (68\%). It needs to be stressed that since medical practitioners could select several options in the questionnaire, different combinations of the above traits were found in the study population.

\section{Clinical progression of the disease}

The severity of ACOS was assessed on the basis of the number of exacerbations treated on an outpatient basis over the past year and on the basis of the number of patients' hospitalizations during their lifetime (Tables 3,4$)$. Exacerbations were found in $68.6 \%$ of the study patients, and in $31.4 \%$ the disease had a stable course, without clinically diagnosed exacerbations. The patients experiencing exacerbations had a mean of 2.11 \pm 1.76 exacerbations over last 12 month of observation. The study patients had a mean of nearly 4 hospitalizations during their lifetime (3.82 \pm 3.67 hospital stays).

One of the main aims of the study was to determine the incidence and types of concomitant diseases in ACOS patients. In the population analyzed in the study reported here, concomitant diseases were diagnosed in $85 \%$ of the patients. The majority of the study's participants had a more than one disease from the list given below (mean number: 2.6). The most common disease was arterial hypertension (62.9\% of the patients), followed by collectively analyzed metabolic disorders (diabetes, obesity,
Table 4. Hospitalizations in the group of patients with asthma-COPD overlap syndrome

\begin{tabular}{cc}
\hline $\begin{array}{c}\text { Patient } \\
\text { was hospitalized once a year }\end{array}$ & $\begin{array}{c}\text { Patient } \\
\text { was not hospitalized }\end{array}$ \\
\hline $56.4 \%$ & $43.6 \%$ \\
\hline Hospitalization statistics & \\
\hline Mean & 3.82 \\
\hline Median & 3 \\
\hline Dominant & 2 \\
\hline Standard deviation & 3.67 \\
\hline
\end{tabular}

metabolic syndrome $-46.4 \%$ of patients), allergic rhinitis (35.7\%), ischaemic heart disease (28\%) and heart failure (13.9\%). Reflux disease was also found in a significant proportion of the patients (21.4\%) (Figure 4).

\section{Most common treatment regimens}

Because of a relatively severe or complicated course of the disease, as much as $94.6 \%$ of the study's patients received multiple inhaled therapy, and only $5.4 \%$ were treated by monotherapy (inhaled glucocorticosteroids (ICS), short-acting $\beta_{2}$-agonists (SABA), long-acting $\beta_{2}$ agonists (LABA) or ipratropium bromide). Multiple therapy regimens were dominated by triple-drug regime, less frequent choice were two medicines or four drugs. Triple-drug regimens usually comprised ICS and LABA (from single or separate inhalers) and cholinolytics (ipratropium bromide and tiotropium bromide). The most commonly used double-drug combination was ICS plus LABA. In quadrupledrug combinations, the added drug was usually theophylline, and in quintuple-drug regimens the typical addition was SABA. Several percent of the study's patients took as many as six drugs. These regimens usually comprised two LABA preparations, concurrent LABA and SABA therapy or concurrent tiotropium and ipratropium bromide treatment. Data collected on the "currently used treatment" applied to treatment regimens in use before specialist consultation and so, in many cases, therapies prescribed by physicians previously treating the patients (i.e. general practitioners and other medical specialists) (Figure 5).

\section{Discussion}

Asthma-chronic obstructive pulmonary disease overlap syndrome is a major clinical problem in outpatient medical practice and has been attracting a lot of attention. Patients with ACOS present with more symptoms, and their severity is greater. Also, they experience more frequent exacerbations and their general condition deteriorates faster. Finally, ACOS patients are more frequently hospitalized [6-9]. Moreover, the self-rated health in ACOS patients is considerably worse than in patients suffering from asthma or COPD diagnosed separately [10]. The costs of medical care provided to ACOS patients 


\begin{tabular}{|lc|}
\hline \multicolumn{2}{|c|}{ Incidence of concomitant diseases } \\
\hline Yes & $85 \%$ \\
\hline No & $15 \%$ \\
\hline
\end{tabular}

Type of concomitant disease

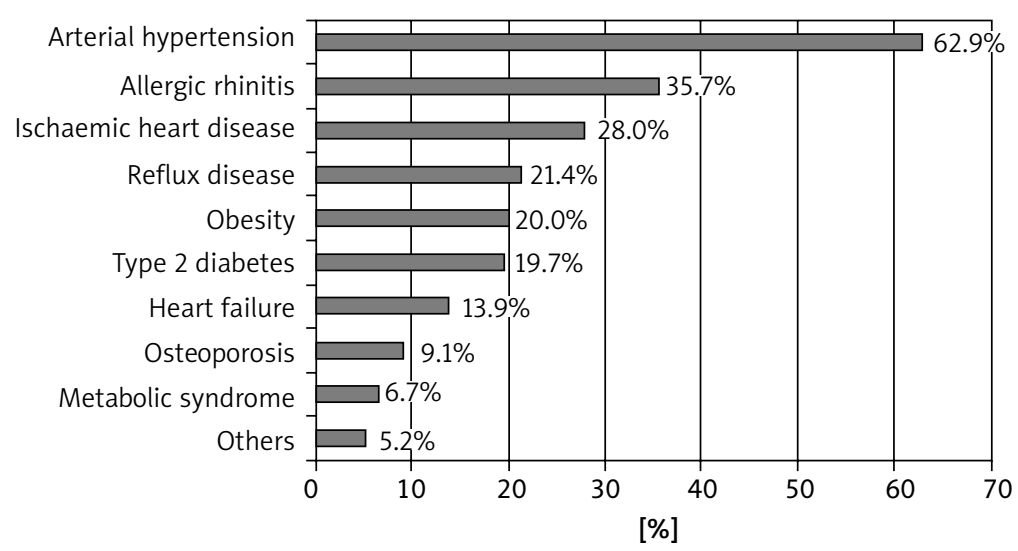

The category of others had the following replies: nervous system diseases (depression, neurosis, schizophrenia), thyroid diseases, gout, history of cerebral stroke, degenerative joint disease.

Figure 4. Concomitant diseases (multiple response chart) $(n=12,103)$

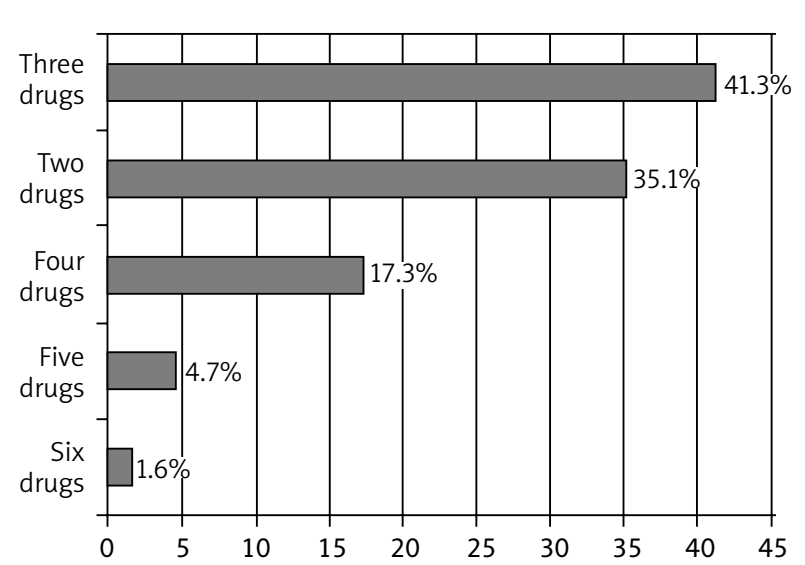

Figure 5. Drug combinations used by patients with asthmaCOPD overlap syndrome

(medication, consultations and hospitalizations) are also twice as high as the costs associated with medical care in typical COPD $[8,11]$. It seems that a broader awareness of ACOS, i.e. a syndrome with a high risk of severe course of bronchial obstructive disease, can and should contribute to a greater individualization of treatment offered to these patients and, consequently, to an improvement of their health status.

The fact that it was so easy for the pulmonary specialists participating in the study reported here to gather such a large population of ACOS patients clearly shows that a combination of asthma and COPD is a common condition. According to available studies, the incidence of ACOS in the general population is ca. 2.4\% (for comparison, the incidence of asthma is 5.6\%, COPD - 7.6\%) [12].
Among the patients diagnosed with bronchial obstructive disease (asthma or COPD), ACOS accounts for $15-25 \%$ of cases. In the population treated by medical specialists, however, the proportion can even reach 55\%. Another factor to consider is that the incidence of ACOS increases along with the age of the patients [7,9]. Due to the generally recognized criterion for the COPD population, i.e. age over 45 years, the study reported here enrolled patients at an age of 46 years or older, which is why the mean age in the study group was 61.5 years. No increase in the incidence of ACOS diagnosis along with age was observed. Quite the opposite: the highest proportion of ACOS patients was aged 56-65 (Figure 1). Approximately a half of the study group were women, which is worth stressing due to the prevalence of the smoking habit among women and the severe course of the overlap syndrome in the female population, particularly in cases of obstructive disease coexisting with obesity.

The main underlying cause of COPD in Poland is smoking, though other factors can also be implicated, such as exposure to products of biomass combustion or recurrent respiratory infections. The same circumstances are involved in the development of COPD in patients with previously diagnosed bronchial asthma or other allergic diseases. In view of the above, one of the enrolment criteria adopted for the study reported here was smoking (100\% of the study patients). It is worthwhile to pay attention to the considerable intensity of the smoking habit (over $28 \pm 13$ pack-years) observed in the followed-up group, even though a considerable proportion of these patients were previously diagnosed with asthma (!). Another important finding was that a very high proportion of the patients continued smoking de- 
spite being diagnosed with bronchial obstructive disease (asthma or COPD), concomitant diseases, and having to follow a complex inhalation therapy regimen $(42.6 \%$ of the study population were still smokers).

Asthma-chronic obstructive pulmonary disease overlap syndrome does not yet have well-defined diagnostic criteria. The first document concerning the syndrome, i.e. the consensus statement published by the Spanish Society for Pulmonology and Thoracic Surgery [4], was a result of discussions held by experts who agreed on the so-called major and minor criteria for diagnosing ACOS in COPD patients. The major criteria included positive bronchodilator response (increase in $\mathrm{FEV}_{1} \geq 15 \%$ and $\geq 400 \mathrm{ml}$ ), sputum eosinophilia and positive history of asthma, and the minor criteria: elevated total IgE, history of atopy and positive bronchodilator response (increase in $\mathrm{FEV}_{1} \geq 12 \%$ and $\geq 200 \mathrm{ml}$ ) in at least two tests. According to the Spanish guidelines, ACOS can be diagnosed if a patient satisfies two major criteria or one major and two minor criteria. The criteria, however, are neither specific, nor sufficiently sensitive. It must be emphasized that the proposed criteria represent a statement agreed by experts, and have not in any way been validated in a population of ACOS patients. Nevertheless, the Spanish criteria must still be acknowledged as a set of guidelines which currently best reflect the clinical phenotype of patients - as opposed to other relatively general criteria, such as GINA.

The joint statement by GINA and GOLD set out in GINA guidelines published on 6 May 2014 [5] is a much "softer" position which does not provide any specific criteria for diagnosing the syndrome. Even though the document enumerates the most typical clinical and spirometric criteria for asthma and COPD, the final diagnosis is left to the physicians, with only a statement that a "similar number" of overlapping features common to asthma and COPD supports the diagnosis of the syndrome.

As opposed to GINA guidelines, which do not prioritize the diagnostic criteria in terms of their importance or incidence, and only enumerate them in an academic sequence, and unlike the Spanish consensus, which specifies major and minor criteria based on an agreement reached by a group of experts, the Polish study is the first one providing a gradation of ACOS features depending on their incidence in the study population and, at the same time, selecting the preferred traits which are the most easily identifiable by pulmonologists providing specialist consultations to patients. Probably due to the latter aspect, the diagnostic criteria for the asthma component and the COPD component are dominated by practical elements of diagnosis (focused on the nature of symptoms, course of the disease, response to treatment), whereas laboratory criteria such as the lgE concentration, blood and sputum eosinophilia, imaging tests, cardiac ultrasound or even the percentage of obstruction reversibility determined by spirometry are only used in asthma and COPD diagnosis as secondary diagnostic elements.
As a preliminary evaluation demonstrates, such "hard" results of additional tests are positioned much higher according to the consensus statements than the daily medical practice in Poland would suggest.

According to specialists, the most commonly enumerated criteria for diagnosing the asthma component include the paroxysmal and variable nature of symptoms (paroxysmal dyspnoea with wheezing) and good response to ICS (diagnosis ex iuvantibus). Other criteria include evidence for allergies and atopy (positive skin-test results, family histories, other allergic conditions in the same patient) and early-onset bronchial disease. Each of these traits was found in over $40 \%$ of the patients. The paroxysmal nature of the disease and its early onset are recognized by GINA among the most important criteria for the diagnosis of the asthma component in ACOS. However, spirometrically confirmed reversibility of obstruction, which is highly prioritized by GINA and the Spanish consensus in the diagnosis of the asthma component, is not widely used by Polish specialists as an obligatory criterion (41.8\% of the patients).

The elements of diagnosis suggesting COPD which were the most common in the study population described here include persistent obstruction continuing after the administration of a bronchodilator (a generally accepted condition for COPD diagnosis), as well as chronic productive cough and progressive exertional dyspnoea. Elements of emphysema and hyperinflation identified in additional (imaging or functional) tests came later down the list: they were found less frequently. These data demonstrate that the COPD component of ACOS is probably closer to the clinical picture of chronic bronchitis. The observation is corroborated by recent reports of a smaller proportion of emphysema and a larger proportion of bronchial wall thickening in ACOS patients in relation to patients with "pure" COPD [13].

\section{Exacerbations and hospitalizations}

All publications dealing with ACOS place an emphasis on the severe course of the syndrome [1-6]. The more severe course is a result of persistent bronchial obstruction on the one hand, and the tendency for paroxysmal deterioration of pulmonary function (the asthma component and bronchial hyperresponsiveness) on the other. Asthma-COPD overlap syndrome is additionally complicated by frequent and severe exacerbations, some of which require hospitalization. According to literature data, ACOS exacerbations are more frequent than in typical COPD and, furthermore, they more often run a severe course [6]. Based on some statistics, ACOS exacerbations are three times more common than in other forms of bronchial obstructive disease. Also, they are associated with greater mortality rates and much higher costs of therapy $[8,11]$. In the reported study, over $68.6 \%$ of the patients represented the frequent-exacerbation phenotype: the mean number of exacerbations treated on an outpatient 
basis during the past year alone was $2.11 \pm 1.76$. The ACOS patients also need more frequent hospitalizations - only less than a half (43.6\%) of the study group had not required hospitalization during their lifetime. The remaining patients, who needed hospital treatment, had been hospitalized at least three times during their lifetime (the mean value; the maximum number: over 30 times).

\section{Concomitant diseases}

Another ACOS-related problem, which requires a more in-depth analysis going beyond the framework of this study, concerns concomitant diseases [12]. According to the study reported here, they accompany ACOS in $85 \%$ of the patients. Such a high incidence of concomitant diseases may be due to a multitude of causes, however the major ones include the smoking habit (a mean of 28 pack-years), age of the patients (a mean of 61.5 years), history of treatment of respiratory diseases (including corticotherapy) and probably lower physical activity levels related to the respiratory disease, and systemic inflammation (recent reports stress that ACOS is associated with a high level of IL- 6 in the blood, correlating with the development of bronchial obstruction and cardiovascular disease) [14].

The main concomitant disease in the study population was arterial hypertension (62\% of the patients), followed by ischaemic heart disease (28\%) and heart failure (13.9\%). Cardiovascular disease is definitely linked to the smoking habit. In the study reported here, ECG or X-ray evidence of chronic pulmonary heart syndrome was only found in $17.5 \%$ of the study patients.

In the context of the selection of an inhaled steroid (ICS - indicated by GINA as first-line treatment in asthma and ACOS with a significant asthma component), it is appropriate to stress the high incidence of metabolic diseases. Obesity, metabolic syndrome or diabetes were found in the analyzed population in over $46 \%$ of the patients.

On account of metabolic diseases or osteoporosis, the treatment of bronchial disease in this group of patients should avoid the use of drugs potentially interfering with their metabolic and hormonal balance, i.e. systemic corticosteroids. This is why ACOS patients should not be treated with oral or parenteral steroids during exacerbation periods. The aspect of safety of ACOS patients definitely supports early and careful selection of an inhaled steroid which, on the one hand, can potentially decrease the number of exacerbations and on the other has a much better safety profile than oral corticosteroids. Even the group of ICS comprises drugs which have a favourable safety profile and minimize potential dangers (i.e. the risk of pneumonia in COPD, increased glucose concentration, decreased production of endogenous cortisol), and such medications (i.e. ciclesonide) should be considered for therapy.

The treatment of patients with a simultaneous diagnosis of asthma and COPD in the study group was based on typically available inhaled preparations from the group of $\beta_{2}$-agonists, cholinolytics and inhaled steroids. In some (probably more challenging) cases, the patients additionally received oral theophylline. Also, the use of two $\beta_{2}$-agonists and the treatment with two cholinolytics concurrently was found. The questionnaire used in the study was simple and did not offer the possibility of correlating the choice of therapy with clinical traits presented by the patients or the severity of their disease.

Despite the publication of several documents, guidelines for the treatment of ACOS are practically nonexistent. The situation can be attributed to the fact that routine clinical trials required for drug approval, according to requirements laid down by different authorities (FDA, EMA), have so far enrolled patients fulfilling strictly defined criteria of "pure" asthma or "pure" COPD. Consequently, ACOS patients have never been subject to controlled observations to determine the efficacy of treatment. A number of experts have recently drawn attention to that fact, stressing that "pure" forms of asthma or COPD are a result of artificial selection, and probably account only for a small percentage of cases requiring long-term treatment of bronchial obstructive disease (!).

In view of the above, the joint statement by GINA and GOLD fails to set out clear guidelines for the treatment of COPD. It only specifies that due to the asthma component the first-line treatment is ICS (at doses adjusted to the severity of the disease), and the COPD component requires combined individualized bronchodilator therapy. The way of selecting therapy for ACOS must be based on clinical considerations resulting from the course of the disease to date, assessment of a patient's response to treatment, diagnosis of concomitant diseases (or their absence) and close monitoring of the efficacy and tolerance of prescribed treatment. The problem of ACOS can be a step forward in the direction of increasingly personalized modern treatment of bronchial obstructive disease involving bronchodilator medicines of different types or anti-inflammatory drugs (inhaled steroids, roflumilast) depending on the patient's phenotype $[15,16]$. In practice, this would mean a thorough revision of the current guidelines for treating both asthma and COPD, which should be regarded as a syndrome of heterogeneous obstructive disorders differing in their pathogenesis, course and, above all, response to different drug classes.

The forthcoming years will see the publication of studies containing more precise treatment guidelines, however before that, more accurate criteria for the diagnosis of ACOS must be established. The recurrent question is: will the British or the Dutch approach prevail? In fact, both are likely to be incorporated.

\section{References}

1. Ghebre MA, Bafadhel M, Desai D, et al. Biological clustering supports both "Dutch" and "British" hypotheses of asthma and chronic obstructive pulmonary disease. J Allergy Clin 
Immunol 2014; pii: S0091-6749(14)00951-8. doi: 10.1016/ j.jaci.2014.06.035.

2. Hardin M, Silverman EK, Barr RG, et al. COPDGene Investigators. The clinical features of the overlap between COPD and asthma. Respir Res 2011; 12: 127.

3. Garcia-Aymerich J, Gomez FP, Benet M, et al. Identification and prospective validation of clinically relevant chronic obstructive pulmonary disease(COPD) subtypes. Thorax 2011; 66: 430-7.

4. Soler-Cataluna JJ, Cosío B, Izquierdo JL, et al. Consensus Document on the overlap phenotype COPD-asthma in COPD. Arch Bronconeumol 2012; 48: 331-7.

5. Global Strategy of Asthma Management and Prevention. Revised 2014. Chapter 5. Diagnosis of asthma, COPD and asthma-COPD overlap syndrome (ACOS). A joint project of GINA and GOLD. www.ginasthma.com

6. Louie S, Zeki AA, Schivo M, et al. The asthma-chronic obstructive pulmonary disease overlap syndrome: pharmacotherapeutic considerations. Expert Rev Clin Pharmacol 2013; 6: 197-219.

7. de Marco R, Pesce G, Marcon A, et al. The coexistence of asthma and chronic obstructive pulmonary disease (COPD): prevalence and risk factors inyoung, middle-aged and elderly people from the general population. PLoS One 2013; 8: 62985.

8. Papaiwannou A, Zarogoulidis P, Porpodis K, et al. Asthmachronic obstructive pulmonary disease overlap syndrome (ACOS): current literature review. J Thorac Dis 2014; 6 (Suppl. 1): S146-51.

9. Zeki AA, Schivo M, Chan A, et al. The asthma-COPD overlap syndrome: a common clinical problem in the elderly. J Allergy (Cairo) 2011; 2011: 861926.

10. Chung JW, Kong KA, Lee JH, et al. Characteristics and selfrated health of overlap syndrome. Int J COPD 2014; 9: 795804.

11. Rhee $\mathrm{CK}$, Yoon $\mathrm{HK}$, Yoo $\mathrm{KH}$, et al. Medical utilization and cost in patients with overlap syndrome of chronic obstructive pulmonary disease and asthma. COPD 2014; 11: 163-70.

12. Pleasants RA, Ohar JA, Croft JB, et al. Chronic obstructive pulmonary disease and asthma - patient characteristics and health impairment. COPD 2014; 11: 256-66.

13. Hardin M, Cho M, McDonald ML, et al. The clinical and genetic features of COPD-asthma overlap syndrome. Eur Respir J 2014; 44: 341-50.

14. Fu JJ, McDonald VM, Gibson PG, Simpson JL. Systemic inflammation in older adults with asthma-COPD overlap syndrome. All Asthma Immunol Res 2014; 6: 316-24.

15. Montuschi P, Malerba M, Santini G, Miravitlles M. Pharmacological treatment of chronic obstructive pulmonary disease: from evidence-based medicine to phenotyping. Drug Discov Today 2014 Aug 23: pii:S1359-6336(14)00331-6. Doi:10.1016/j.drudis.2014.08.004 [Epub ahead of print].

16. Miravittles M, Soler-Cataluńa JJ, Calle M, Soriano JB. Treatment of COPD by clinical phenotypes: putting old evidence into clinical practice. Eur Respir J 2013; 41: 1252-6. 